\title{
EDITORIAL
}

For reprint orders, please contact: reprints@futuremedicine.com

\section{How can we achieve HPV control in Europe?}
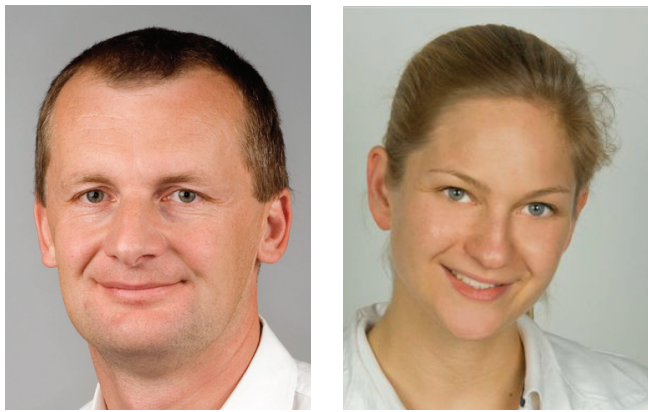

\author{
"More than 44,000 new cases \\ of HPV-related anogenital \\ cancers affect men and \\ women in Europe annually.”
}

Elmar A Joura*,1 \& Sophie Pils ${ }^{1}$

\section{How can we achieve HPV control in Europe?}

The upcoming year 2016 will be marked by two significant events: the ninevalent HPV vaccine will be available in Europe and The Netherlands will be the first country to implement primary HPV screening for the precursors of cervical cancer.

Europe has a substantial burden of HPV-related disease. More than 44,000 new cases of HPV-related anogenital cancers affect men and women in Europe annually [1]. In addition, more than 15,000 new cases of mainly HPV-16related oropharyngeal cancer affect predominantly males. The estimation of genital precancer is in the order of 300,000 or more annual cases. Genital warts are a substantial limitation in the sexual wellbeing; probably more than 800,000 new cases are diagnosed in European females and males every year [2].

In 2006, a quadrivalent HPV 6/11/16/18 vaccine $\left(\right.$ Gardasil ${ }^{\circledR}$, Sanofi Pasteur MSD) became available, in 2007 a bivalent HPV 16/18 vaccine was licensed in Europe (Cervarix ${ }^{\circledR}$, GSK) [3] .
These vaccines effectively prevent infections and disease caused by the two most potent oncogenic HPV types. The quadrivalent vaccine also prevents highly efficacious infections with HPV 6/11, causing approximately $90 \%$ of genital warts. Both vaccines have an excellent safety profile [4]. HPV 16 and 18 cause $70 \%$ of HPV-related cervical cancer but only $50 \%$ of cervical precancer. A vaccine covering HPV 16/18/31/33/45/52/58 potentially prevents $85-90 \%$ of cervical precancer, $90 \%$ of invasive cervical cancer and a substantial proportion of other HPV-related cancers $[1,5]$.

In a Phase III study including young women aged $16-26$, the ninevalent vaccine has been shown to prevent $97 \%$ of infections and lesions related to HPV $31 / 33 / 45 / 52 / 58$ with a noninferior immunogenicity for HPV 6/11/16/18 [6]. In June 2015, this vaccine was licensed by EMA for females and males from the age of nine [7]. The immunogenicity has been even superior in boys and girls compared with young women [8]. It can be given concomitantly with other vaccines [9]. With this new vaccine, almost 40,000

\section{KEYWORDS}

- cancer vaccine • cervical cancer screening $\bullet$ human papillomavirus

“For every vaccination program, coverage is key." 
“...the ninevalent vaccine has been shown to prevent $97 \%$ of infections and lesions related to HPV 31/33/45/52/58 with a noninferior immunogenicity for HPV 6/11/16/18." new cancers are potentially preventable annually in the long run, an additional 6000 cases in comparison to the first-generation vaccines [1].

So far only three European countries have achieved a sufficient coverage rate in the target population of adolescents. Denmark, Portugal and the UK have achieved $80 \%$ or more coverage in females, a prerequisite for the building of herd protection. Denmark has provided excellent data on the reduction of genital warts and cervical precancer [10]. In countries with a lower coverage gender-neutral vaccination is an even more important option. The quadrivalent vaccine has been demonstrated to be highly effective in preventing genital warts and anal disease in males [11,12]. A female-only program even with a good coverage leaves men having sex with men unprotected [13]. Austria was the first country worldwide to recommend HPV vaccination for both sexes as early as 2007, and in 2014 the first European country to provide a fully funded gender-neutral HPV vaccination program. This program focuses on the age group of 9-10-year-old children, with a catch-up to the age of 15 and is considered to be cost effective [14]. The young age for vaccination gives the opportunity to apply a twodose schedule which is easier to apply and cost saving [15]. For every vaccination program, coverage is key. Vaccinating at a young age, preferably in a school-based program is most effective, however it remains to be evaluated up to which age such a catch-up program remains to be cost effective. Both first-generation vaccines have demonstrated clinical efficacy up to the age of 45 [16], and even after treatment for HPV-related disease, vaccinated persons have less subsequent disease [17].

Today, we are aware that HPV causes a broad spectrum of disease in both sexes, the prevention of cervical cancer remains a key issue. In eastern Europe, a high incidence is observed, but also in other parts of Europe women are still at risk of dying from this disease. Large trials have demonstrated that HPV testing is not only more sensitive in the detection of cervical precancer, it has also been shown that HPV testing is also superior in preventing invasive cervical cancer in comparison to cytology, a negative test almost excludes invasive disease for more than 5 years [18]. With HPV tests identifying the most important oncogenic types HPV 16 and 18, women at risk can be better stratified and undergo adequate treatment [19].
Other oncogenic HPV types cause cancer later in life, hence in women with non-HPV 16/18 infections conizations can be delayed and preterm deliveries avoided.

In an HPV vaccinated population, the positive predictive value of cytology screening is even lower. This favors primary HPV screening followed by triage cytology. The Netherlands introduces primary HPV testing in 2016, reducing the number of screening rounds to five. For noncompliant women who account for about $30 \%$ of the population and who have a higher risk of cervical cancer, the opportunity of HPV self-testing is offered. It has been demonstrated that this strategy has the potential to further decrease the number of cervical cancers [20]. HPV testing in the urine has shown promising results and may be an option for women reluctant to pelvic examination and even self-manipulation [21].

The future perspective is a sensible and cost effective combination of vaccination and screening. With a ninevalent HPV vaccine most of cervical and other HPV-related cancers can be avoided. A gender-neutral vaccination program at an early age with a broad catchup for adolescents and even adults is currently the most effective way to prevent HPV-related infections and disease, in infants two doses of any HPV vaccine are probably sufficient and even one dose might be a future option [22]. In a nonvaccinated population, HPV testing is superior to the conventional PAP screening; in a vaccinated population it will become mandatory. A transition from the annual PAP smear, which is still practiced in countries like Germany to just a few lifetime HPV tests, has already started and screening algorithms will be adopted. The value of adult vaccination remains to be evaluated in upcoming large-scale trials [23].

Financial \& competing interests disclosure

EA Joura has received research grants through institution from Merck and GSK, advisory board fees from Merck and Sanofi Pasteur MSD and lecture fees from Merck, Sanofi Pasteur MSD, GSK and Roche Diagnostics. The authors have no other relevant affiliations or financial involvement with any organization or entity with a financial interest in or financial conflict with the subject matter or materials discussed in the manuscript apart from those disclosed.

No writing assistance was utilized in the production of this manuscript. 


\section{References}

1 Hartwig S, Baldauf JJ, Dominiak-Felden G et al. Estimation of the epidemiological burden of HPV-related anogenital cancers, precancerous lesions, and genital warts in women and men in Europe: potential additional benefit of a nine-valent second generation HPV vaccine compared with the first generation HPV vaccines. Papillomavirus Res. doi:10.1016/j.pvr.06.003 (2015) (Epub ahead of print).

2 Hartwig S, Syrjänen S, Dominiak-Felden G, Brotons M, Castellsagué X. Estimation of the epidemiological burden of human papillomavirus-related cancers and non-malignant diseases in men in Europe: a review. BMC Cancer 12, 30 (2012).

3 Pils S, Joura EA. From the monovalent to the nine-valent HPV vaccine. Clin. Microbiol. Infect. 21(9), 827-833 (2015).

4 Vichnin M, Bonanni P, Klein NP et al. An overview of quadrivalent human papillomavirus vaccine safety -2006 to 2015 . Pediatr. Infect. Dis. J. 34(9), 983-991 (2015).

5 Joura EA, Ault KA, Bosch FX et al. Attribution of 12 high-risk human papillomavirus genotypes to infection and cervical disease. Cancer Epidemiol. Biomarkers Prev. 23(10), 1997-2008 (2014).

6 Joura EA, Giuliano AR, Iversen OE et al. A 9-valent $\mathrm{HPV}$ vaccine against infection and intraepithelial neoplasia in women. N. Engl. J. Med. 372(8), 711-723 (2015).

7 EMA. www.ema.europa.eu/ema

8 Van Damme P, Olsson SE, Block S et al. Immunogenicity and safety of a 9-valent HPV vaccine. Pediatrics 136(1), e28-e39 (2015).
9 Kosalaraksa P, Mehlsen J, Vesikari T et al. An open-label, randomized study of a 9-valent human papillomavirus vaccine given concomitantly with diphtheria, tetanus, pertussis and poliomyelitis vaccines to healthy adolescents 11-15 years of age. Pediatr. Infect. Dis. J. 34(6), 627-634 (2015).

10 Baldur-Felskov B, Dehlendorff C, Munk C, Kjaer SK. Early impact of humanpapillomavirus vaccination on cervical neoplasia - nationwide follow-up of young Danish women. J. Natl Cancer Inst. 106(3), djt460 (2014).

11 Giuliano AR, Palefsky JM, Goldstone S et al. Efficacy of quadrivalent HPV vaccine against HPV Infection and disease in males. N. Engl. J. Med. 364(5), 401-411 (2011).

12 Palefsky JM, Giuliano AR, Goldstone $S$ et al. HPV vaccine against anal HPV infection and anal intraepithelial neoplasia. N. Engl. J. Med. 365(17), 1576-1585 (2011).

13 Stanley M. HPV vaccination in boys and men. Hum. Vaccin. Immunother. 10(7), 2106-2108 (2014).

14 Bresse X, Goergen C, Prager B, Joura E. Universal vaccination with the quadrivalent HPV vaccine in Austria: impact on virus circulation, public health and cost-effectiveness analysis. Expert Rev. Pharmacoecon. Outcomes Res. 14(2), 269-281 (2014).

15 Stanley MA, Sudenga SL, Giuliano AR. Alternative dosage schedules with HPV virus-like particle vaccines. Expert Rev. Vaccines 13(8), 1027-1038 (2014).

16 Skinner SR, Szarewski A, Romanowski B et al. Efficacy, safety, and immunogenicity of the human papillomavirus 16/18 AS04-adjuvanted vaccine in women older than 25 years: 4 year interim follow-up of the
Phase 3, double-blind, randomised controlled VIVIANE study. Lancet 384(9961), 2213-2227 (2014).

17 Joura EA, Garland SM, Paavonen J et al. Effect of the human papillomavirus (HPV) quadrivalent vaccine in a subgroup of women with cervical and vulvar disease: retrospective pooled analysis of trial data. BMJ 27(344), e1401 (2012).

18 Ronco G, Dillner J, Elfström KM et al. Efficacy of HPV-based screening for prevention of invasive cervical cancer: follow-up of four European randomised controlled trials. Lancet 383(9916), 524-532 (2014).

19 Wright TC, Stoler MH, Behrens CM, Sharma A, Zhang G, Wright TL. Primary cervical cancer screening with human papillomavirus: end of study results from the ATHENA study using HPV as the first-line screening test. Gynecol. Oncol.136(2), 189-197 (2015).

20 Gök M, Heideman DA, van Kemenade FJ et al. Offering self-sampling for human papillomavirus testing to non-attendees of the cervical screening programme: characteristics of the responders. Eur. J. Cancer 48(12), 1799-1808 (2012).

21 Pathak N, Dodds J, Zamora J, Khan K. Accuracy of urinary human papillomavirus testing for presence of cervical HPV: systematic review and meta-analysis. BMJ 16(349), g5264 (2014).

22 Schiller JT, Lowy DR. Raising expectations for subunit vaccine. J. Infect. Dis. 211(9), 1373-1375 (2015)

23 Bosch FX, Robles C, Díaz M et al. HPV-FASTER: broadening the scopefor prevention of HPV-related cancer. Nat. Rev. Clin. Oncol. doi:10.1038/nrclinonc.2015.146 (2015) (Epub ahead of print). 